\title{
BMJ Open Mental health disorders among healthcare workers during the COVID-19 pandemic: a cross-sectional survey from three major hospitals in Kenya
}

\author{
Jasmit Shah, ${ }^{1}$ Aliza Monroe-Wise (D) , ${ }^{2}$ Zohray Talib, ${ }^{3}$ Alphonse Nabiswa, ${ }^{1}$ \\ Mohammed Said, ${ }_{4}^{4}$ Abdulaziz Abeid, ${ }^{5}$ Mohamed Ali Mohamed, ${ }^{5}$ Sood Mohamed, ${ }^{5}$ \\ Sayed K Ali (1D ${ }^{1}$
}

To cite: Shah J, Monroe-Wise A, Talib Z, et al. Mental health disorders among healthcare workers during the COVID-19 pandemic: a cross-sectional survey from three major hospitals in Kenya. BMJ Open 2021;11:e050316. doi:10.1136/ bmjopen-2021-050316

- Prepublication history for this paper is available online. To view these files, please visit the journal online (http://dx.doi org/10.1136/bmjopen-2021 050316).

Received 16 February 2021 Accepted 14 May 2021

Check for updates

(C) Author(s) (or their employer(s)) 2021. Re-use permitted under CC BY-NC. No commercial re-use. See rights and permissions. Published by BMJ.

${ }^{1}$ Department of Internal Medicine, Aga Khan University Hospital, Nairobi, Kenya ${ }^{2}$ Department of Global Health, University of Washington Seattle Campus, Seattle, Washington, USA

${ }^{3}$ Department of Medical Education, California University of Science and Medicine, San Bernardino, California, USA

${ }^{4}$ Avenue Hospital, Nairobi, Kenya ${ }^{5}$ Coast General Teaching and Referral Hospital, Nairobi, Kenya

Correspondence to

Dr Sayed K Ali;

sayed.karar@aku.edu

\section{ABSTRACT}

Background COVID-19 is an international global health emergency and has posed a great challenge to mental well-being and resilience. Little is known about the mental health impact of COVID-19 among healthcare workers (HCWs) in sub-Saharan Africa or other low-resource settings.

Methods We conducted a cross-sectional study between August and November 2020 among HCWs recruited from three major hospitals in Kenya. The survey questionnaire consisted of six components: demographic and work title characteristics; information regarding care of patients with COVID-19; and symptoms of depression, anxiety, insomnia, distress and burnout, measured using standardised questionnaires. Multivariable logistic regression analysis was performed to identify factors associated with mental health disorders.

Results A total of 433 (65.2\% response rate) individuals participated in the survey. Median age was 32.75 years, $58.4 \%$ were females and $68.8 \%$ were front-line workers. Depression, anxiety, insomnia, distress and burnout were reported in $53.6 \%, 44.3 \%, 41.1 \%, 31.0 \%$ and $45.8 \%$ of all participants, respectively. Front-line HCWs, females and doctors were at higher risk of mental health symptoms. Nearly half of participants reported inadequate resources or training to care for patients with COVID-19, and those in the government hospital were more likely to report mental health symptoms.

Conclusions This is among the first studies examining mental health outcomes among HCWs during the COVID-19 pandemic in Kenya. Similar to other studies from around the world, HCWs directly involved with patients with COVID-19 reported higher rates of mental health symptoms. Mitigating strategies specific to Kenyan HCWs are urgently needed to help them cope with mental health symptoms during the pandemic.

\section{BACKGROUND}

The immense toll of the global SARS-CoV-2 pandemic continues to rise, with over 170 million confirmed cases and almost 3.5 million deaths to date. ${ }^{1}$ COVID-19 reached Kenya in March 2020, and as of June 2021,
Strengths and limitations of this study

- To the best of our knowledge, this is one of the first articles reporting the prevalence of mental health disorders among healthcare workers (HCWs) during the COVID-19 pandemic in Kenya.

- Mental health disorders were measured using specific standardised questionnaires.

- The study was conducted between the first and second waves of the virus outbreak and the results might not be suggestive of the long-term psychological disorders within our HCW population.

- There is need for cost-effective mitigating strategies to help curb the burden of mental health disorders associated with caring for patients with COVID-19 in Kenya.

there were over 170000 confirmed cases and 13000 deaths. ${ }^{1}$ COVID-19 has had a significant impact on the healthcare workforce. Increased workload, long hours, inadequate personal protective equipment (PPE), the need to make ethically and morally difficult decisions, a lack of social support, and isolation from and fear of infecting family members and oneself can have major impacts on the mental health of front-line workers. ${ }^{2}$ Multiple recent studies conducted in Turkey, Singapore, India, Italy, Spain, China, the USA and Oman have shown high rates of stress, depression, anxiety and burnout among healthcare workers (HCW) taking care of patients with COVID-19..$^{3-9}$ Other studies have shown higher rates of mental health disorders among HCWs caring for patients with COVID-19 compared with those not caring for patients with COVID-19. ${ }^{10-15}$ Risk factors for mental health symptoms among HCWs caring for patients with COVID-19 include being female, young, single, having minimal work experience and working on the front 
lines. ${ }^{10}$ Unfortunately, the psychological impact of the COVID-19 pandemic has also resulted in HCW suicides. ${ }^{16}$ A global study done between April and May 2020 across 31 countries looking at mental health outcomes in HCWs during the initial stage of the pandemic showed an overall prevalence of $60 \%$ anxiety and $53 \%$ depression. There were only 49 responses from 11 countries in Africa, including Kenya. ${ }^{17}$ This was the beginning of the COVID-19 in most African countries where the cases were still low (Kenya: April 2020 total was $~ 400$ positive cases). ${ }^{1}$

Quality healthcare, especially during a pandemic, requires a robust health workforce with adequate numbers, optimal physical and mental well-being and a supportive environment. Many parts of Africa continue to struggle with the rising cases of COVID-19 amidst limited medical resources and infrastructure, inadequate healthcare workforce and minimal intensive care unit (ICU) beds. The Organisation for Economic Co-operation and Development in 2020 reported 2.6 and 3 physicians and 11.9 and 7.8 nurses per 1000 people in the USA and UK, respectively. Austria reported the most physicians (5.2) while Norway reported the most nurses (18) per 1000 people. ${ }^{18}$ Most recent corresponding figures from the WHO in Kenya were 0.2 physicians and 1.2 nurses per 1000 people. ${ }^{19}$ Additionally, Kenya has approximately 14 hospital beds per 10000 people with 537 ICU beds and 256 ventilators serving a population of close to 50 million people. ${ }^{1920}$ In contrast, the WHO in 2019 reported 25 hospital beds per 10000 people in the UK with a population of approximately 67 million people. ${ }^{19}$ Many African countries have fragile healthcare systems with fewer than 30 critical care beds for their entire population and only a handful of fully trained critical care physicians, highlighting the lack of critical resources to adequately address the COVID-19 pandemic in Africa. ${ }^{21}$ Mental health resources and providers are also lacking in Kenya. As of 2016, Kenya reported 0.18 psychiatrists and 0.002 psychologists per 10000 people with less than $1 \%$ of the public sector having access to any form of mental healthcare services. ${ }^{19}{ }^{22}$ Furthermore, similar to other subSaharan African (SSA) countries, Kenya has no formal mental health response plan within the larger COVID-19 strategic response. ${ }^{22}$

Historical experiences with deadly pandemics can offer invaluable lessons to resource-limited settings. During the Ebola pandemic in Western Africa in the early parts of 2014, the pandemic swiftly crippled an already weak healthcare system, resulting in approximately $69 \%$ of HCW deaths with anecdotal evidence of increasing mental health disorders among survivors. ${ }^{23-25}$ However, few studies have been conducted on the association of the Ebola pandemic on HCW mental health symptoms. Similarly, a year into the COVID-19 pandemic, little is known on the effect of the pandemic on the mental health of HCWs in SSA or in any resource-limited setting. We therefore aimed to measure the prevalence of mental health symptoms among HCWs in three major hospitals in Kenya, and to evaluate risk factors for mental health symptoms among HCWs in these hospitals.

\section{METHODS}

\section{Study design}

We carried out a cross-sectional study between August and November 2020.

\section{Participants and study site}

Physicians, including residents and fellows, and nurses were recruited from one government (Coast General Teaching and Referral Hospital, Mombasa (CGTRH)) and two private hospitals (Aga Khan University Hospital, Nairobi (AKUHN) and Avenue Hospital, Nairobi).

\section{Recruitment and enrolment}

Email addresses of all physicians and nurses in each hospital were obtained by the principal site investigator in each hospital with help of the Information Technology Department. Physicians and nurses were recruited from AKUHN, CGTRH and Avenue Hospital. Email invitations with a link to a voluntary, deidentified survey were sent to 725 HCWs. Responses from HCWs remained anonymous.

\section{Procedures}

Online survey data were collected through the Research Electronic Data Capture (REDCap) platform (Vanderbilt and National Institute of Health) ${ }^{26}$ Email reminders were sent out twice a week from REDCap for participation in the survey. Every participant received a unique link allowing them to complete the survey only once. The survey questionnaire in English consisted of six components: demographic characteristics (including job title, experiences providing care to patients with COVID19), and sections on symptoms of depression, anxiety, insomnia, distress and burnout. On average, the surveys took about 5-7 min to complete. Mental health symptoms were measured using validated questionnaires: the 9-item Patient Health Questionnaire (PHQ-9) for depression, ${ }^{27}$ the 7-item Generalized Anxiety Disorder Questionnaire (GAD-7) for anxiety, ${ }^{28}$ the 7 -item Insomnia Severity Index Questionnaire (ISI) for insomnia, ${ }^{29}$ the 22-item Impact of Event Scale-Revised (IES-R) for distress caused by traumatic events ${ }^{30}$ and the 16-item Stanford Professional Fulfillment Index Questionnaire (SPFI) for burnout. ${ }^{31}$ While the 22-item IES-R was initially designed for diagnosing post-traumatic stress disorder, it has been used in multiple recent studies to measure COVID-19-related psychological distress and we have used it to measure general distress caused by traumatic events. ${ }^{3} 13$ The cutoff score for detecting symptoms of major depression, anxiety, insomnia and distress was $10,7,15$ and 26, respectively. ${ }^{13}$ The categories of the severity of each mental health disorder are presented in table 1 . Based on previously published literature, we defined front-line HCWs as those HCWs who reported in providing direct care (diagnosing, treating or providing nursing care) as 'frontline HCWs' to patients with COVID-19. ${ }^{13} 15$ HCWs who were not taking care of patients with COVID-19 were mostly from the outpatient clinics as well as the non-COVID-19 wards. 
Table 1 Scoring categories for the mental health disorders

\begin{tabular}{ll} 
PHQ-9 (Depression) & IES-R (Distress) \\
\hline Normal (0-4) & Normal (0-8) \\
\hline Mild (5-9) & Mild (9-25) \\
Moderate (10-14) & Moderate (26-43) \\
Severe (15-27) & Severe (44-88) \\
Binary cut-off-10 & Binary cut-off-26 \\
GAD-7 (Anxiety) & SPFI (Burnout) \\
Normal (0-4) & Yes (>1.33) \\
Mild (5-9) & No ( $\leq 1.33)$ \\
Moderate (10-14) & \\
Severe (15-21) & SPFI (Professional fulfilment) \\
Binary cut-off-7 & Yes (>3.0) \\
ISI (Insomnia) & No ( $\leq 3.0)$ \\
Normal (0-7) & \\
Subthreshold (8-14) & \\
Moderate (15-21) & \\
Severe (22-28) & \\
Binary cut-off-15 & \\
\hline
\end{tabular}

GAD-7, 7-item Generalized Anxiety Disorder; IES-R, Impact of Event Scale-Revised; ISI, Insomnia Severity Index; PHQ-9, 9item Patient Health Questionnaire; SPFI, Stanford Professional Fulfillment Index.

\section{Statistical analysis}

Categorical data were analysed as frequencies and percentages whereas continuous data were analysed as medians with IQRs. The non-parametric Kruskal-Wallis test was used to compare the continuous variables and Fisher's exact test was used to compare the categorical variables between two groups. To determine potential risk factors for symptoms of depression, anxiety, insomnia, distress, burnout and professional fulfilment in participants, a multivariate logistic regression analysis was performed, and the associations between risk factors and outcomes are presented as ORs and 95\% CIs. The outcome variables for the multivariate logistic regressions were mental health disorders dichotomised using the standard cut-offs as above. Data analysis was performed using SPSS statistical software V.20.0 (IBM). The significance level was set at $\alpha=0.05$, and all tests were two tailed.

\section{Patient and public involvement}

Patients or the public were not involved in the research design, reporting or survey dissemination strategies of this study.

\section{RESULTS}

Of the 725 invited HCWs across the three hospitals, 473 (65.2\%) responded to the survey and 433 (91.5\%, overall: $59.7 \%$ ) consented to complete the survey (table 2). The median age of the participants was 32.8 years (IQR: 29.7,
Table 2 Baseline characteristics of study participants $(\mathrm{n}=433)$

\begin{tabular}{|c|c|}
\hline Characteristics & n (\%) \\
\hline Age (years) $(n=401)$ (median [IQR]) & $32.75(29.71,36.75)$ \\
\hline \multicolumn{2}{|l|}{ Gender } \\
\hline Male & $176(40.6)$ \\
\hline Female & $253(58.4)$ \\
\hline Prefer not to disclose & $4(1.0)$ \\
\hline \multicolumn{2}{|l|}{ Marital status $(n=430)$} \\
\hline Single & $172(40.0)$ \\
\hline Married & $244(56.7)$ \\
\hline Other & $14(3.3)$ \\
\hline \multicolumn{2}{|l|}{ Ethnicity $(\mathrm{n}=429)$} \\
\hline African & $354(82.5)$ \\
\hline Asian & $51(11.9)$ \\
\hline Other & $24(5.6)$ \\
\hline \multicolumn{2}{|l|}{ Healthcare profession } \\
\hline Nurses & $190(43.9)$ \\
\hline Doctors & $243(56.1)$ \\
\hline \multicolumn{2}{|l|}{ Direct COVID-19 care } \\
\hline Yes & $298(68.8)$ \\
\hline No & $135(31.2)$ \\
\hline \multicolumn{2}{|l|}{ Patients cared $(n=297)$} \\
\hline$<5$ patients & $101(34.0)$ \\
\hline 5-20 patients & $122(41.1)$ \\
\hline$>20$ patients & $74(24.9)$ \\
\hline \multicolumn{2}{|l|}{ Lost any patients $(\mathrm{n}=296)$} \\
\hline Yes & $174(58.8)$ \\
\hline No & $122(41.2)$ \\
\hline \multicolumn{2}{|c|}{ Enough resources to care for COVID-19 $(n=297)$} \\
\hline Yes & $161(54.2)$ \\
\hline No & $136(45.8)$ \\
\hline \multicolumn{2}{|c|}{ Adequately trained for COVID-19 $(n=298)$} \\
\hline Yes & $161(54.0)$ \\
\hline No & $137(46.0)$ \\
\hline \multicolumn{2}{|l|}{ History of mental health disorder } \\
\hline Yes & $16(3.70)$ \\
\hline No & $398(91.9)$ \\
\hline Not sure & $15(3.4)$ \\
\hline Prefer not to disclose & $4(1.0)$ \\
\hline \multicolumn{2}{|l|}{ Diagnosis $(n=15)$} \\
\hline Anxiety & $5(33.3)$ \\
\hline Depression & $7(46.7)$ \\
\hline Epilepsy & $1(6.7)$ \\
\hline Depression and anxiety & $2(13.3)$ \\
\hline \multicolumn{2}{|l|}{ Still have symptoms } \\
\hline Yes & $8(50.0)$ \\
\hline No & $8(50.0)$ \\
\hline
\end{tabular}


$36.8)$. Of the 433 responding participants, 253 (58.4\%) were females, and 244 (56.7\%) were married. Most of the participants were medical doctors $(243(56.1 \%))$ and of African ethnicity (354 (82.5\%)). A total of 298 participants $(68.8 \%)$ were front-line HCWs directly engaged in COVID-19 care and treatment. Of the front-line workers, $122(41.1 \%)$ had cared for about 5-20 patients and 174 (58.8\%) had lost a patient to COVID-19. Only $3.7 \%$ of all participants reported a history or diagnosis of any mental health disorder.

Using the standardised screening tools, depression, anxiety, insomnia, distress and burnout were reported in $53.6 \%, 44.3 \%, 41.1 \%, 31.0 \%$ and $45.8 \%$ of all participants, respectively. Severe symptoms of depression, anxiety, insomnia, distress and burnout were more commonly reported among front-line HCWs than colleagues who were not front-line HCWs. For example, severe depression was reported among $11.6 \%$ of front-line HCWs $(n=34)$ compared with $3.8 \%(n=5)$ among workers not directly caring for patients with COVID-19 ( $<<0.001$, table 3$)$. The questionnaires demonstrated good internal consistency with a Cronbach's alpha of 0.904, 0.912, 0.896, 0.971 and 0.935 for PHQ-9, GAD-7, ISI, IES-R and SPFI, respectively.

Multivariate logistic regression analysis showed that after adjustment, working on the front lines was an independent risk variable for all the mental health disorders (depression: OR 3.55, 95\% CI 1.77 to $7.11, \mathrm{p}<0.001$; anxiety: OR 2.51, 95\% CI 1.20 to $5.25, \mathrm{p}=0.014$; insomnia: OR 4.45, 95\% CI 1.51 to $13.10, \mathrm{p}=0.007$; distress: OR 1.89 , 95\% CI 1.10 to 3.17, $\mathrm{p}=0.021$; burnout: OR 2.74, $95 \%$ CI 1.69 to $4.42, \mathrm{p}<0.001)$. Furthermore, being a doctor and being female were associated with severe symptoms of depression and anxiety, with an OR of 1.95 for severe depression among women compared with men $(95 \%$ CI 1.12 to $3.42 ; p=0.019$ ) and an OR of 2.29 for severe anxiety among doctors compared with nurses (95\% CI 1.21 to 4.32; $\mathrm{p}=0.011$ ) (table 4). Although age was not statistically associated with dichotomised mental health outcomes in multivariate analysis, higher age was correlated with lower mental health disorder scores by Pearson's correlation. The correlation for age with depression, anxiety, insomnia, distress and burnout was $-0.144,-0.118,-0.179$, -0.144 and -0.239 , respectively (all $\mathrm{p}<0.001$ ).

Nearly half of front-line workers stated that they lacked adequate resources and PPE to care for patients with COVID-19, and fewer felt that they needed additional training to take care of or treat patients with COVID-19 (resources: $45.8 \%$, trained: $46.0 \%$ ). On further analysis, we found that HCWs employed in public institutions were more likely to report having inadequate resources than those in the private hospital (OR 14.55, 95\% CI 5.99 to $35.34 ; \mathrm{p}<0.001)$, and were more likely to report being inadequately trained (OR 3.61, 95\% CI 1.91 to 6.81; $\mathrm{p}<0.001)$.

Furthermore, respondents from the public institution reported experiencing more moderate to severe symptom levels of depression, anxiety, insomnia and distress (eg, moderate and severe depression among private vs public:
$67(18.7 \%)$ vs $22(32.4 \%), \mathrm{p}=0.014$; moderate and severe anxiety among private vs public: $43(12.3 \%)$ vs 19 (28.3\%), $\mathrm{p}=0.008$; moderate and severe insomnia among private vs public: 30 (8.5\%) vs 15 (22.4\%), $\mathrm{p}=0.004$; distress among private vs public: $62(17.8 \%)$ vs $21(33.3 \%), \mathrm{p}=0.014)$ (table 5).

\section{DISCUSSION}

To our knowledge, this is among the first studies to investigate mental health disorders among HCWs during the COVID-19 pandemic in Kenya, or to evaluate the associations between COVID-19 and mental health symptoms in any resource-limited setting in SSA. Our study examined HCWs in one government and two private hospitals in Kenya actively involved in the care of patients with COVID-19. Two of the institutions were located in Nairobi and one in Mombasa; both major cities have been greatly affected by COVID-19.

Despite only 3.7\% reporting ever having mental health disorders previously, our study reported higher rates of depression, anxiety, insomnia, distress and burnout. Similar to studies conducted in other countries, being a front-line worker was associated with higher scores for depression, anxiety, insomnia, distress and burnout. Our study also showed that being female and being a doctor were significantly associated with severe symptoms of depression and anxiety. This is in contrast to other studies in which nurses experienced more psychological symptoms associated with caring for patients with COVID19. ${ }^{5} 32$ This specific finding needs further research to understand if there is a cultural aspect related to this and whether level of knowledge and education plays a role in the anxiety level of different HCWs.

The median age of our study population was 32.8 years. Age was significantly negatively correlated with mental health disorders which was similar to findings from other studies. ${ }^{11} 1533$ We observed higher overall percentages of depression, anxiety and insomnia among frontline HCWs taking care of patients with COVID-19 than have been found in similar studies conducted in higher income countries using the same validated scales and diagnostic criteria. ${ }^{3611-15}$ While methods of these studies vary slightly, this finding in our setting could be attributed to the limited resources and lack of mitigating strategies such as a formal mental health response plan, readily available mental health services, regulation of work hours and adequate medical resources in Kenya.

A recent study on mental health disorders among trainees at three American academic medical centres found that trainees taking care of patients with COVID-19 had higher rates of burnout compared with those not taking care of patients with COVID-19, but found no significant difference in professional fulfilment scores. ${ }^{34}$ On the contrary, a study in Turkey showed a lower level of burnout in physicians taking care of patients with COVID-19 compared with physicians who took care of patients without COVID-19. ${ }^{35}$ In our study, we found 
Table 3 Severity categories and scores of depression, anxiety, insomnia, distress, burnout and professional fulfilment measurements in total cohort and categorised between direct COVID-19 care

\begin{tabular}{|c|c|c|c|c|c|}
\hline & \multirow[b]{2}{*}{ Total } & \multicolumn{3}{|c|}{ Have you ever directly cared for a patient with COVID-19? } \\
\hline & & & Yes & No & $P$ value \\
\hline \multicolumn{2}{|l|}{ Depression } & $(n=425)$ & $(n=292)$ & $(n=133)$ & $<0.001$ \\
\hline \multirow[t]{4}{*}{ PHQ-9 } & None & $197(46.4 \%)$ & $116(39.7 \%)$ & $81(60.9 \%)$ & \\
\hline & Mild & $139(32.7 \%)$ & 99 (33.9\%) & $40(30.1 \%)$ & \\
\hline & Moderate & $50(11.8 \%)$ & $43(14.7 \%)$ & $7(5.3 \%)$ & \\
\hline & Severe & $39(9.2 \%)$ & $34(11.6 \%)$ & $5(3.8 \%)$ & \\
\hline \multicolumn{2}{|l|}{ Anxiety } & $(n=418)$ & $(n=287)$ & $(n=131)$ & 0.017 \\
\hline \multirow[t]{4}{*}{ GAD-7 } & Minimal & $208(49.8 \%)$ & $131(45.6 \%)$ & $77(58.8 \%)$ & \\
\hline & Mild & $148(35.4 \%)$ & $104(36.2 \%)$ & $44(33.6 \%)$ & \\
\hline & Moderate & $33(7.9 \%)$ & $27(9.4 \%)$ & $6(4.6 \%)$ & \\
\hline & Severe & $29(6.9 \%)$ & $25(8.7 \%)$ & $4(3.1 \%)$ & \\
\hline \multicolumn{2}{|l|}{ Insomnia } & $(n=418)$ & $(n=287)$ & $(n=131)$ & 0.001 \\
\hline \multirow[t]{4}{*}{ ISI } & None & $246(58.9 \%)$ & $156(54.4 \%)$ & $90(68.7 \%)$ & \\
\hline & Subthreshold & $127(30.4 \%)$ & $90(31.4 \%)$ & $37(28.2 \%)$ & \\
\hline & Moderate & $39(9.3 \%)$ & $36(12.5 \%)$ & $3(2.3 \%)$ & \\
\hline & Severe & $6(1.4 \%)$ & $5(1.7 \%)$ & $1(0.8 \%)$ & \\
\hline \multicolumn{2}{|l|}{ Distress } & $(n=410)$ & $(n=281)$ & $(n=129)$ & 0.008 \\
\hline \multirow[t]{4}{*}{ IES-R } & Normal & $283(69.0 \%)$ & $185(65.8 \%)$ & $98(76.0 \%)$ & \\
\hline & Mild & $44(10.7 \%)$ & $28(10.0 \%)$ & $16(12.4 \%)$ & \\
\hline & Moderate & $24(5.9 \%)$ & $17(6.0 \%)$ & $7(5.4 \%)$ & \\
\hline & Severe & 59 (14.4\%) & $51(18.1 \%)$ & $8(6.2 \%)$ & \\
\hline \multicolumn{2}{|l|}{ Burnout } & $(n=404)$ & $(n=278)$ & $(n=126)$ & \\
\hline & $\leq 1.33$ & $219(54.2 \%)$ & $130(46.8 \%)$ & $89(70.6 \%)$ & $<0.001$ \\
\hline & $>1.33$ & $185(45.8 \%)$ & $148(53.2 \%)$ & $37(29.4 \%)$ & \\
\hline \multicolumn{6}{|c|}{ Professional fulfilment } \\
\hline & $\leq 3.00$ & $327(81.1 \%)$ & $231(83.1 \%)$ & $96(76.8 \%)$ & 0.168 \\
\hline & $>3.00$ & $76(18.9 \%)$ & 47 (16.9\%) & $29(23.2 \%)$ & \\
\hline \multicolumn{6}{|c|}{ IES-R subscales } \\
\hline \multicolumn{2}{|c|}{ Avoidance subscale } & $0.80(0.10,1.40)$ & $0.90(0.10,1.80)$ & $0.60(0.00,1.10)$ & 0.032 \\
\hline \multicolumn{2}{|c|}{ Intrusion subscale } & $0.65(0.10,1.30)$ & $0.80(0.10,1.50)$ & $0.60(0.00,1.10)$ & 0.065 \\
\hline \multicolumn{2}{|c|}{ Hyperarousal subscale } & $0.40(0.00,1.20)$ & $0.50(0.20,1.30)$ & $0.30(0.00,0.80)$ & 0.041 \\
\hline \multicolumn{6}{|c|}{ Burnout subscales } \\
\hline \multicolumn{2}{|c|}{ Professional fulfilment } & $54.17(37.50,75.00)$ & $50.00(33.33,75.00)$ & $58.33(45.83,75.00)$ & $<0.001$ \\
\hline \multicolumn{2}{|c|}{ Work exhaustion } & $43.75(25.00,68.75)$ & $50.00(25.00,75.00)$ & $31.25(18.75,50.00)$ & $<0.001$ \\
\hline \multicolumn{2}{|c|}{ Interpersonal disengagement } & $20.42(0.00,37.50)$ & $25.00(0.00,41.67)$ & $8.33(0.00,25.00)$ & $<0.001$ \\
\hline
\end{tabular}

GAD-7, 7-item Generalized Anxiety Disorder; IES-R, Impact of Event Scale-Revised; ISI, Insomnia Severity Index; PHQ-9, 9-item Patient Health Questionnaire.

statistically higher rates of burnout among all cadres of HCWs at all institutions taking care of patients with COVID-19 compared with those not taking care of patients with COVID-19. We found no statistical difference in professional fulfilment scores.

Overall, we found that half of the respondents felt inadequately trained and reported lack of enough resources and PPE to safely care for patients with COVID-19. A study looking at HCW knowledge, attitude and practices in Uganda found that $69 \%$ reported sufficient knowledge and $74 \%$ had a positive attitude around COVID-19. ${ }^{36}$ Similarly, Mbachu and colleagues reported excellent knowledge and good preventative practices towards COVID-19 in Nigeria. ${ }^{37}$

In our study, HCWs at the government hospital felt less trained with inadequate resources and PPE to take 
Table 4 Multivariate logistic regression analysis

\begin{tabular}{|c|c|c|c|c|c|c|c|}
\hline Variable & Adjusted OR* & $95 \% \mathrm{Cl}$ & $P$ value & Variable & Adjusted OR* & $95 \% \mathrm{Cl}$ & $P$ value \\
\hline \multicolumn{4}{|c|}{ PHQ-9: Depression } & \multicolumn{4}{|l|}{ IES-R: Distress } \\
\hline \multicolumn{4}{|l|}{ Gender } & \multicolumn{4}{|l|}{ Gender } \\
\hline Female & 1.95 & 1.12 to 3.42 & 0.019 & Female & 2.18 & 1.32 to 3.60 & 0.002 \\
\hline \multicolumn{4}{|l|}{ Healthcare } & \multicolumn{4}{|l|}{ Healthcare } \\
\hline Doctor & 2.24 & 1.27 to 3.94 & 0.005 & Doctor & 1.09 & 0.67 to 1.76 & 0.738 \\
\hline \multicolumn{4}{|l|}{ COVID-19 care } & \multicolumn{4}{|l|}{ COVID-19 care } \\
\hline Second line & 1 (Reference) & & N/A & Second line & 1 (Reference) & & $\mathrm{N} / \mathrm{A}$ \\
\hline Front line & 3.55 & 1.77 to 7.11 & $<0.001$ & Front line & 1.89 & 1.10 to 3.17 & 0.021 \\
\hline \multicolumn{4}{|l|}{ GAD-7: Anxiety } & \multicolumn{4}{|l|}{ SPFI: Burnout } \\
\hline Female & 1.95 & 1.04 to 3.65 & 0.036 & Female & 1.73 & 1.11 to 2.71 & 0.017 \\
\hline \multicolumn{4}{|l|}{ Healthcare } & \multicolumn{4}{|l|}{ Healthcare } \\
\hline Nurse & 1 (Reference) & & N/A & Nurse & 1 (Reference) & & N/A \\
\hline Doctor & 2.29 & 1.21 to 4.32 & 0.011 & Doctor & 0.86 & 0.55 to 1.34 & 0.858 \\
\hline \multicolumn{4}{|l|}{ COVID-19 care } & \multicolumn{4}{|l|}{ COVID-19 care } \\
\hline Second line & 1 (Reference) & & N/A & Second line & 1 (Reference) & & $\mathrm{N} / \mathrm{A}$ \\
\hline Front line & 2.51 & 1.20 to 5.25 & 0.014 & Front line & 2.74 & 1.69 to 4.42 & $<0.001$ \\
\hline \multicolumn{4}{|l|}{ ISI: Insomnia } & \multicolumn{4}{|c|}{ SPFI: Professional fulfilment } \\
\hline \multicolumn{4}{|l|}{ Gender } & \multicolumn{4}{|l|}{ Gender } \\
\hline Second line & 1 (Reference) & & N/A & Second line & 1 (Reference) & & $\mathrm{N} / \mathrm{A}$ \\
\hline Front line & 4.45 & 1.51 to 13.1 & 0.007 & Front line & 0.78 & 0.45 to 1.37 & 0.788 \\
\hline
\end{tabular}

*Adjusted for age, gender, marital status, working title and COVID-19 care where appropriate.

GAD-7, 7-item Generalized Anxiety Disorder; IES-R, Impact of Event Scale-Revised; ISI, Insomnia Severity Index; N/A, not applicable; PHQ-9, 9-item Patient Health Questionnaire; SPFI, Stanford Professional Fulfillment Index.

care of patients with COVID-19 than those in the private hospitals. Fifty-two per cent of the hospitals in Kenya are funded by the government. These public hospitals frequently operate with inadequate healthcare workforce and basic amenities, with 74 of 2927 primary public hospitals $(2.5 \%)$ reporting lacking a medical doctor to serve the local population ${ }^{38}$ and only $55 \%$ of the healthcare facilities having basic amenities including sanitation facilities, communication technologies, consultation rooms, improved water sources, power supplies, emergency rooms and computers with internet access. Furthermore, only $12 \%$ of health facilities in Kenya report having items necessary for infection prevention. ${ }^{38}$ Even though the private sector owns and manages almost two-thirds of all Kenya's healthcare facilities, basic data on healthcare resources are lacking. ${ }^{39} 40$ A report from USAID Kenya Private Health Sector Assessment highlights the inequalities of healthcare resources in Kenya with three-quarters of doctors and approximately two-thirds of nurses and clinical officers employed by the private sector. ${ }^{39}$ In addition, compared with the public sector, the private sector offers better remuneration, minimising HCW strikes and improving the overall work environment. ${ }^{40}$ COVID-19 has significantly contributed to many unresolved logistical issues in public hospitals, including waste management, infrastructure and funding, affecting the morale and welfare of HCWs. ${ }^{41}$ Other recent studies have shown that challenges in acquisition of PPE in public hospitals are 
Table 5 Severity categories of depression, anxiety, insomnia, distress, burnout and professional fulfilment measurements in total cohort and categorised between private and public hospitals

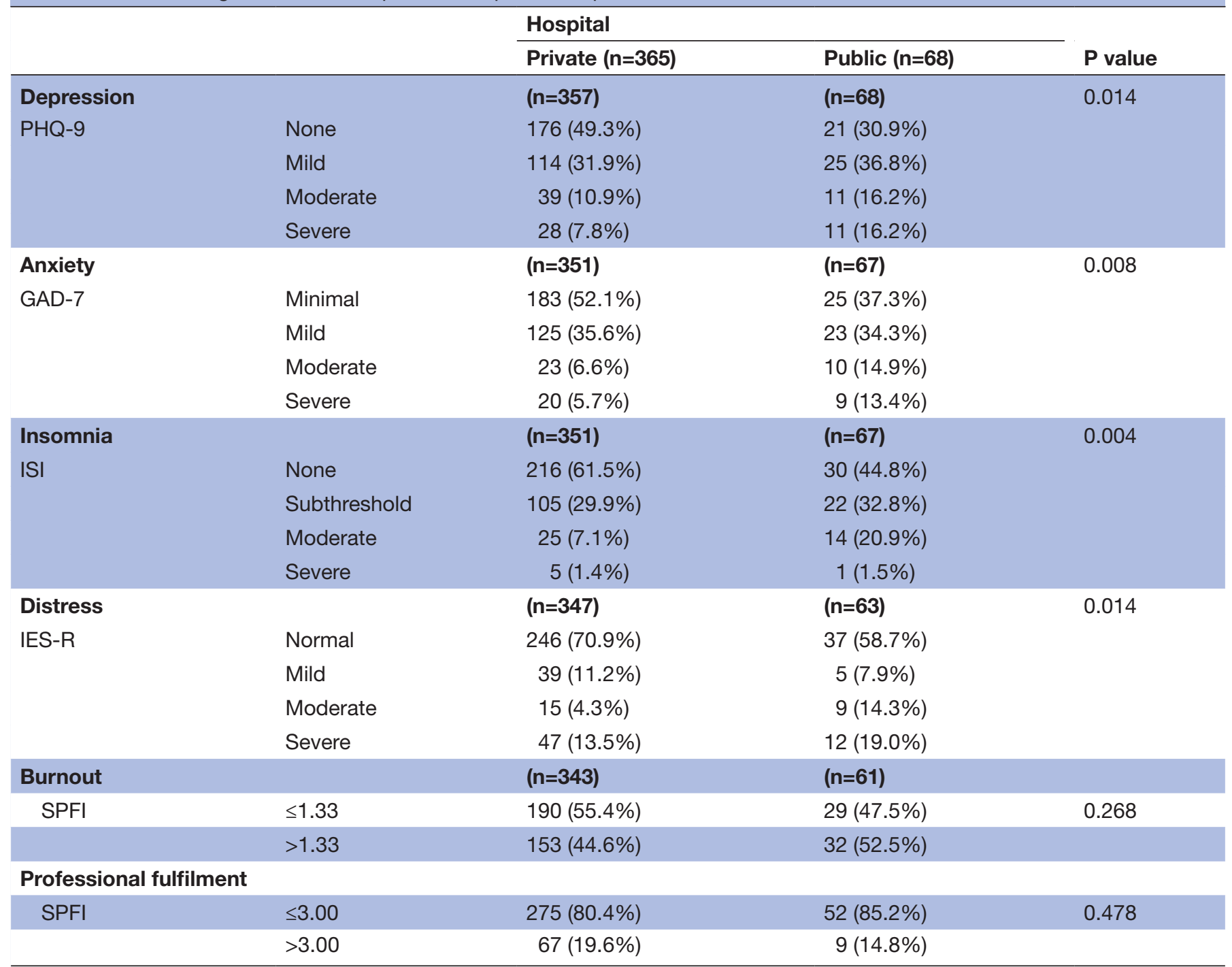

GAD-7, 7-item Generalized Anxiety Disorder; IES-R, Impact of Event Scale-Revised; ISI, Insomnia Severity Index; PHQ-9, 9-item Patient Health Questionnaire; SPFI, Stanford Professional Fulfillment Index.

associated with higher levels of anxiety for both clinical and support staff. ${ }^{41}$ Mbachu and colleagues showed that a lack of PPE had a negative impact on the attitudes of HCWs in Nigeria. ${ }^{37}$ Likewise, in our study, HCWs in the government institution also showed significantly higher rates of depression, anxiety, insomnia and distress than those in the private hospitals. We could find no other study comparing mental health outcomes between private and government hospitals in our setting or any other settings. In addition to the absence of mitigating strategies unique to HCWs taking care of patients with COVID19 , our findings also stress the importance of adequate medical personnel, training, critical care beds and other key resources to help fortify fragile healthcare systems within many African countries and to better tackle the COVID-19 pandemic.

Like many other countries in SSA, Kenya lacks a formal mental health response plan to the COVID-19 pandemic. ${ }^{22}$ Some researcher in Kenya has called for key recommendations to help strengthen the mental health response to COVID-19 including a dynamic mental health response plan designed by mental health experts specific to the needs of the country and grass-roots training of community health workers and volunteers, especially HIV counsellors and retired nurses, remains key to target the population at large and to focus on providing key mental health services to HCWs in institutions across the country. ${ }^{22}$ Like many other African countries, regular press releases on television and radios are used to disseminate key information on COVID-19 in Kenya. ${ }^{42}$ These platforms can be used to educate the population on COVID-19 but also to demystify the stigma around seeking mental health services related to COVID19. Of note, Kenya has a $91 \%$ penetration of mobile subscriptions allowing the use of mobile health applications to further enhance the delivery of care to the local 
population and to HCWs. ${ }^{22}$ In addition, toll-free mental health helplines could be a key step to improving access to mental health services especially for specific populations such as HCWs providing care to patients with COVID-19. ${ }^{42}$ Senior leadership at every hospital, with the support of the government and donor organisations, should seek creative ways to enhance the mental health capabilities and resources especially for HCWs taking care of patients with COVID-19. This includes either investing in training and capacity-building opportunities or sharing resources among various hospitals geographically and electronically to better support the response process. Providing daily allowances to front-line HCWs, additional tax relief, as well as publicly acknowledging their heroic efforts might be ways to boost morale and improve mental health outcomes in resource-limited settings. ${ }^{2}$

Our study has several limitations. It was conducted in one government hospital in Mombasa and two private hospitals in Nairobi, with different funding structures. This was a cross-sectional survey and the responses were not equally distributed among the HCW cadres, which could bias the results. This may have occurred due to higher email utilisation among doctors than among nurses. Also being a cross-sectional survey, the study could only describe the prevalence of mental health symptoms. Our study was conducted between the first and second waves of the virus outbreak and our results might not be suggestive of the long-term psychological disorders within our HCW population. We also did not ask the participants if they had contracted COVID-19 themselves or if they had unprotected exposure to COVID-19. Electronic mail with frequent reminders was only used, which could have potentially limited our response rate. Lastly, there may have been a response bias in that the non-responders may have been suffering from mental health symptoms, limiting the motivation to respond.

\section{CONCLUSION}

Our study, the first conducted in Kenya and one of the first in a low-resource healthcare setting, shows markedly higher rates of mental health disorders including depression and anxiety in all HCWs taking care of patients with COVID-19 than those not caring for patients with COVID19 , and higher rates than those reported in higher income countries. Being a doctor and being female were associated with severe symptoms of depression and anxiety. Furthermore, HCWs in the government institution experienced higher rates of mental health symptoms than their counterparts in private institutions. This study highlights the urgent need for cost-effective, easy-to-replicate and rapid-to-implement mitigating strategies to help curb the burden of mental health disorders associated with caring for patients with COVID-19 in Kenya and other resource-limited countries. Special attention should be paid to HCWs in the government hospitals.
Contributors SKA developed the study concept and proposal, supervised the overall project and coordinated the study at AKUHN. JS, AMW, ZT and AN assisted in the study concept, design and editing of the survey. MS supervised data collection at the Avenue Hospital. AA, MAM and SM supervised the data collection at CGTRH. SKA and JS verified the underlying data. JS did the data analysis. JS and SKA wrote the first draft of the paper. All authors reviewed, edited and provided comments on the first and subsequent drafts. All authors reviewed and approved the final manuscript.

Funding The authors have not declared a specific grant for this research from any funding agency in the public, commercial or not-for-profit sectors.

Competing interests None declared.

Patient consent for publication Not required.

Ethics approval Approval for this study was obtained from the Institutional Ethics and Review Committee at the Aga Khan University, Nairobi and the hospital leadership at CGTRH and Avenue Hospital. Online consent was obtained from all the participants. Participants were allowed to withdraw from the study at any time without any consequences. The survey was anonymous, and confidentiality of information was assured. On conclusion of the survey, a score for each mental health disorder was computed and shared with the participants. If the scores indicated a mental health issue, the participants were directed to seek medical consultation with their primary care provider or the counselling services at the respective hospitals. If any of the participants indicated suicidal or homicidal ideations, they were directed to the mental health hot line at the Aga Khan University Hospital or an assigned physician at the CGTRH and Avenue Hospital.

Provenance and peer review Not commissioned; externally peer reviewed.

Data availability statement Data are available upon reasonable request. The data for this study are available from the corresponding author on reasonable request.

Open access This is an open access article distributed in accordance with the Creative Commons Attribution Non Commercial (CC BY-NC 4.0) license, which permits others to distribute, remix, adapt, build upon this work non-commercially, and license their derivative works on different terms, provided the original work is properly cited, appropriate credit is given, any changes made indicated, and the use is non-commercial. See: http://creativecommons.org/licenses/by-nc/4.0/.

\section{ORCID iDs}

Aliza Monroe-Wise http://orcid.org/0000-0002-8843-3462

Sayed K Ali http://orcid.org/0000-0003-0750-2903

\section{REFERENCES}

1 World Health Organization. WHO coronavirus disease (COVID-19) Dashboard. Available: https://covid19.who.int/ [Accessed 9 Jan 2021].

2 Chersich MF, Gray G, Fairlie L, et al. COVID-19 in Africa: care and protection for frontline healthcare workers. Global Health 2020;16:46.

3 Chew NWS, Lee GKH, Tan BYQ, et al. A multinational, multicentre study on the psychological outcomes and associated physical symptoms amongst healthcare workers during COVID-19 outbreak. Brain Behav Immun 2020;88:559-65.

4 Shechter A, Diaz F, Moise N, et al. Psychological distress, coping behaviors, and preferences for support among New York healthcare workers during the COVID-19 pandemic. Gen Hosp Psychiatry 2020;66:1-8.

5 Pappa S, Ntella V, Giannakas T, et al. Prevalence of depression, anxiety, and insomnia among healthcare workers during the COVID-19 pandemic: a systematic review and meta-analysis. Brain Behav Immun 2020;88:901-7.

6 Bohlken J, Schömig F, Lemke MR, et al. [COVID-19 Pandemic: Stress Experience of Healthcare Workers - A Short Current Review]. Psychiatr Prax 2020;47:190-7.

7 Martínez-López José Ángel, Lázaro-Pérez C, Gómez-Galán J. Psychological impact of COVID-19 emergency on health professionals: Burnout incidence at the most critical period in Spain. $J$ Clin Med 2020;9:3029.

8 Liu X, Chen J, Wang D, et al. COVID-19 outbreak can change the job burnout in health care professionals. Front Psychiatry 2020;11:563781.

9 Khasne RW, Dhakulkar BS, Mahajan HC, et al. Burnout among healthcare workers during COVID-19 pandemic in India: results of a questionnaire-based survey. Indian J Crit Care Med 2020;24:664-71.

10 Elbay RY, Kurtulmuș A, Arpacıoğlu S, et al. Depression, anxiety, stress levels of physicians and associated factors in Covid-19 pandemics. Psychiatry Res 2020;290:113130. 
11 Rossi R, Socci V, Pacitti F, et al. Mental health outcomes among frontline and second-line health care workers during the coronavirus disease 2019 (COVID-19) pandemic in Italy. JAMA Netw Open 2020;3:e2010185.

12 Evanoff BA, Strickland JR, Dale AM, et al. Work-Related and personal factors associated with mental well-being during the COVID-19 response: survey of health care and other workers. $J$ Med Internet Res 2020;22:e21366.

13 Lai J, Ma S, Wang Y, et al. Factors associated with mental health outcomes among health care workers exposed to coronavirus disease 2019. JAMA Netw Open 2020;3:e203976.

14 Zhang W-R, Wang K, Yin L, et al. Mental health and psychosocial problems of medical health workers during the COVID-19 epidemic in China. Psychother Psychosom 2020;89:242-50.

15 Badahdah A, Khamis F, Al Mahyijari N, et al. The mental health of health care workers in Oman during the COVID-19 pandemic. Int $J$ Soc Psychiatry 2020:002076402093959.

16 Watkins A, Rothfeld M, Rashbaum WK. Top E.R. doctor who treated virus patients dies by suicide. New York Times, 2020.

17 Htay MNN, Marzo RR, AlRifai A, et al. Immediate impact of COVID-19 on mental health and its associated factors among healthcare workers: a global perspective across 31 countries. J Glob Health 2020;10:020381.

18 OECD. OECD health indicators, OECD. Available: https://data.oecd. org/health.htm [Accessed 9 Jan 2021].

19 World Health Organization. The global health Observatory, 2020. Available: http://www.who.int/hrh/statistics/hwfstats/

20 Barasa EW, Ouma PO, Okiro EA. Assessing the hospital surge capacity of the Kenyan health system in the face of the COVID-19 pandemic. PLoS One 2020;15:e0236308.

21 Murthy S, Leligdowicz A, Adhikari NKJ. Intensive care unit capacity in low-income countries: a systematic review. PLoS One 2015;10:e0116949.

22 Jaguga F, Kwobah E. Mental health response to the COVID-19 pandemic in Kenya: a review. Int J Ment Health Syst 2020;14:68.

23 Kamara S, Walder A, Duncan J, et al. Mental health care during the Ebola virus disease outbreak in Sierra Leone. Bull World Health Organ 2017;95:842-7.

24 International Medical Corps. Assessment of mental health and psychosocial support (MHPSS) needs and resources in the context of Ebola, 2014. Available: http://ebolacommunicationnetwork.org/ wp-content/uploads/2015/01/IMC-Sierra-Leone-Dec-2014-EbolaMHPSS-Assessment.pdf [Accessed 9 Jan 2021].

25 World Health Organization. Health worker Ebola infections in guinea, Liberia and Sierra Leone, 2015. Available: https://www.who.int/hrh/ documents/21may2015_web_final.pdf [Accessed 9 Jan 2021].

26 Harris PA, Taylor R, Minor BL, et al. The REDCap Consortium: building an international community of software platform partners. J Biomed Inform 2019;95:103208.

27 Kroenke K, Spitzer RL, Williams JB. The PHQ-9: validity of a brief depression severity measure. J Gen Intern Med 2001;16:606-13.
28 Spitzer RL, Kroenke K, Williams JBW, et al. A brief measure for assessing generalized anxiety disorder: the GAD-7. Arch Intern Med 2006;166:1092-7.

29 Morin CM, Belleville G, Bélanger L, et al. The insomnia severity index: psychometric indicators to detect insomnia cases and evaluate treatment response. Sleep 2011;34:601-8.

30 Motlagh H. Impact of event Scale-revised. J Physiother 2010;56:203

31 Trockel M, Bohman B, Lesure E, et al. A brief instrument to assess both burnout and professional fulfillment in physicians: reliability and validity, including correlation with self-reported medical errors, in a sample of resident and practicing physicians. Acad Psychiatry 2018;42:11-24

32 Tan BYQ, Chew NWS, Lee GKH, et al. Psychological impact of the COVID-19 pandemic on health care workers in Singapore. Ann Intern Med 2020;173:317-20.

33 Youssef N, Mostafa A, Ezzat R, et al. Mental health status of health-care professionals working in quarantine and non-quarantine Egyptian hospitals during the COVID-19 pandemic. East Mediterr Health J 2020;26:1155-64.

34 Kannampallil TG, Goss CW, Evanoff BA, et al. Exposure to COVID-19 patients increases physician trainee stress and burnout. PLOS One 2020;15:e0237301.

35 Dinibutun SR. Factors associated with burnout among physicians: an evaluation during a period of COVID-19 pandemic. $J$ Healthc Leadersh 2020;12:85-94.

36 Olum R, Chekwech G, Wekha G, et al. Coronavirus Disease-2019: knowledge, attitude, and practices of health care workers at Makerere university teaching hospitals, Uganda. Front Public Health 2020;8:181.

37 Mbachu CNP, Azubuike CM-C, Mbachu II, et al. COVID-19 infection: knowledge, attitude, practices, and impact among healthcare workers in a south-eastern Nigerian state. J Infect Dev Ctries 2020;14:943-52.

38 Ministry of Health. The Kenya harmonized health facility assessment (KHFA), 2018. Available: https://www.health.go.ke/wp-content/ uploads/2020/01/KHFA-2018-19-Popular-version-report-Final-.pdf

39 United States Agency for Interntional Development. Kenya private health sector assessment, 2009. Available: https://www. shopsplusproject.org/sites/default/files/resources/FINAL1\% 20KPHSA\%20Country\%20Report.pdf

40 Embassy of the Kingdom of the Netherlands. Kenyan Healthcare Sector: Opportunities for the Dutch Life Sciences \& Health Sector, 2016. Available: http://khf.co.ke/wp-content/uploads/2018/03/2016Kenyan-Healthcare-Sector-Report.pdf

41 Maina M, Tosas-Auguet O, English M, et al. Infection prevention and control during the COVID-19 pandemic: challenges and opportunities for Kenyan public hospitals. Wellcome Open Res 2020;5:211.

42 Semo B-werq, Frissa SM. The Mental Health Impact of the COVID-19 Pandemic: Implications for Sub-Saharan Africa]]\&gt. Psychol Res Behav Manag 2020;13:713-20. 